УДК 51-74

\title{
Математическое описание процесса теплообмена между потоками га- за и дисперсного материала
}

\author{
А. В. Солодкая ${ }^{1}$ И. Л. Боикова \\ Одесская национальная академия пищевых технологий, ул. Канатная, 112, Одесса, 65039, Украина \\ e-mail: ${ }^{1}$ a solodkaya@mail.ru, ${ }^{2}$ ira boshkova@mail.ru \\ ORCID: ${ }^{1}$ http://orcid.org/0000-0002-4043-7667, ${ }^{2}$ http://orcid.org/0000-0001-5989-9223
}

\begin{abstract}
Анализируются преимущества применения теплообменников контактного типа и существующие подходы к математическому представлению теплообмена между газом и частицами. Представлена математическая модель теплообмена между движущиимся плотным слоем дисперсного материала и потоком газа. Получены аналитические зависимости для расчета локальных температур газового и твердого компонентов. Представлены результаты расчетов температур компонентов по высоте канала для прямоточной и противоточной схемы движения.
\end{abstract}

Ключевые слова: Теплообменник; Дисперсный материал; Прямоток; Противоток; Математическая модель; Температура компонентов

\section{Математичний опис процеса теплообміну між потоками газу та дисперсного матеріалу}

\author{
А. В. Солодка ${ }^{1}$, І. Л. Бошкова
}

Одеська національна академія харчових технологій, вул. Канатна, 112, м. Одеса, 65039, Україна

e-mail: 1a_solodkaya@mail.ru, ${ }^{2}$ ira_boshkova@mail.ru

ORCID: ${ }^{1}$ http://orcid.org/0000-0002-4043-7667, ${ }^{2}$ http://orcid.org/0000-0001-5989-9223

\begin{abstract}
Аналізуються переваги застосування теплообмінників контактного типу та існуючі підходи до математичного уявлення теплообміну між газом і частками. Представлена математична модель теплообміну між щзільним рухомим шаром дисперсного матеріалу та потоком газу. Отримані аналітичні залежності для розрахунків локальних температур газового і твердого компонентів. Представлені результати розрахунків температур компонентів по висоті каналу для прямоточної і протитечійної схеми руху.
\end{abstract}

Ключові слова: Теплообмінник; Дисперсний матеріал; Прямоток; Протитечія; Математична модель; Температура компонентів.

DOI: http://dx.doi.org/10.15673/ret.v53i2.593

(C) The Author(s) 2017. This article is an open access publication

This work is licensed under the Creative Commons Attribution 4.0 International License (CC BY)

http://creativecommons.org/licenses/by/4.0/

\section{1 Введение}

Интерес к конструированию теплообменников контактного типа, особенностями которых является передача теплоты путем непосредственного соприкосновения рабочих тел, обусловлен рядом их преимуществ в сравнении с поверхностными теплообменниками [10], [11], [12]. К основным преимуществам теплообменников такого типа относится высокая интенсивность процессов теплообмена, существенное уменьшение коррозии оборудования, исключение возможности отложений на поверхностях нагрева, возможность повышения температурного уровня технологических процессов, простота конструкции и снижение затрат дефицитных материалов и, соответственно, материальных затрат; отсутствие разделяющей поверхности, что позволяет использовать в качестве теплоносителей загрязненные и агрессивные газы, жидкости, высококонцентрированные растворы. Эти преимущества определили широкую сферу применения контактных теплообменников. В данной работе предметом аналитического исследования является теплообменник для утилизации теплоты отходящих дымовых газов, в котором в качестве нагреваемого теплоносителя используется движущейся в виде плотного слоя дисперсный материал,

Моделированию процессов теплообмена между газом и твердыми частицами уделяется значительное внимание [1], [2], [3] вследствие важности знаний о влиянии отдельных факторов и условий на распределение температур и эффективность теплообмена. В [4] предложена ячеечная математическая модель теплообмена между стохастически движущимися потоками газа 
и сыпучего материала при распределенной по длине подаче горячего газа, Модель применима для противои прямотока и позволяет получать данные по распределению температур газового и твердого компонентов в теплообменном аппарате. Предложенная модель представляет безусловный научный интерес для контактных теплообменников с распределенной подачей газа, однако для расчета технологически важных характеристик процесса и выработки рекомендации по его рациональной организации необходимо максимальное приближение условий однозначности к рассматриваемой частной задаче. В работах [5] моделированию процессов теплообмена в сочетании с массообменом и при учете протекания химических реакций между потоками газового и твердого компонентов уделяется значительное внимание. Полученные решения позволяют определять поля температур и влагосодержаний для аппаратов с плотными слоевыми дисперсными системами, применяемыми в энергетике, металлургии, химической, пищевой и других отраслях промышленности. Наиболее результативными представляются двухкомпонентные гомогенные модели, в которых слой рассматривается как система, состоящая из двух квазисплошных компонентов, между которыми протекают межкомпонентные процессы теплообмена [5, 6, 7, 8, 9].

\section{2 Аналитическое исследование теплообмена между потоками газа и плотного слоя частиц}

Для получения аналитическим путем информации о распределении температур в контактном теплообменнике, одним из теплоносителей в котором является дисперсный материал, разработаны математические модели теплообмена между твердыми частицами и газовой (воздушной) средой, движущимися по схеме прямотока и противотока. Схема участка теплообмена для противоточного движения теплоносителей представлена на рисунке 1 .

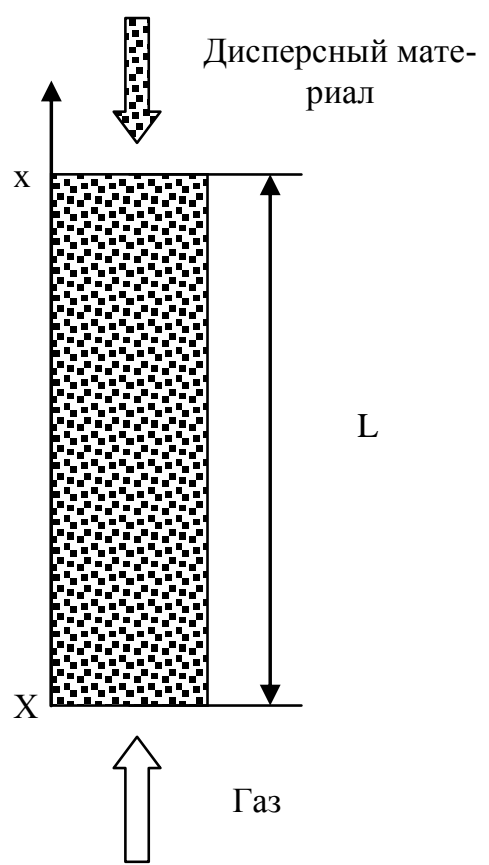

Рисунок 1 - Схема участка теплообмена между потоками газа и дисперсного материала.
Газовый (воздушный) поток подавался снизу $(x=0)$, дисперсный материал поступает в теплообменный участок сверху $(x=L)$.

Тепломассоперенос в контактном теплообменнике для одномерной задачи описывается следующей системой уравнений:

- уравнение энергии для газового компонента

$$
c_{2} \rho_{2} \varepsilon \frac{\partial t_{2}}{\partial \tau}=\lambda_{2}^{*} \frac{\partial^{2} t_{2}}{\partial x_{2}^{2}}-c_{2} \rho_{2} w_{2}^{\prime \prime} \frac{\partial t_{2}}{\partial y}-\alpha_{M} a_{y \partial}\left(t_{2}-t_{m}\right) \text {; }
$$

- уравнение энергии для твердого компонента

$$
\begin{aligned}
& c_{m} \rho_{m}(1-\varepsilon) \frac{\partial t_{m}}{\partial \tau}=\lambda_{m}^{*} \frac{\partial^{2} t_{m}}{\partial x_{2}^{2}}-c_{m} \rho_{m}(1-\varepsilon) w_{m}^{\prime \prime} \frac{\partial t_{m}}{\partial x}- \\
& -\alpha_{M} a_{y \partial}\left(t_{2}-t_{m}\right)
\end{aligned}
$$

Здесь $c_{2}, c_{m}$ - удельная теплоемкость газа и материала соответственно, $\rho_{2}, \rho_{m}-$ плотность газа и твердого компонента соответственно, $w_{m}^{\prime \prime}$ - скорость слоя сыпучего материала в аппарате, $a_{y д}$ - удельная поверхность частиц в единице объема слоя, $\left(\mathrm{M}^{2} / \mathrm{M}^{3}\right)$, зависящая от их размера, формы и порозности слоя:

$$
\mathrm{a}_{y \partial}=\frac{6(1-\varepsilon)}{d_{\ni}},
$$

где $d_{\text {э }}$ - эквивалентный диаметр частиц, $\varepsilon-$ порозность слоя. Порозность плотного движущегося слоя $\varepsilon$ изменяется в пределах 0.32...0.43.

$$
d_{\ni}=1,24 \cdot \sqrt[3]{\frac{m_{m}}{\rho_{m}}}
$$

где $m_{m}-$ масса одной частицы. $\alpha_{м}-$ коэффициент межкомпонентного теплообмена между газом и частицами материала, $\mathrm{BT} /\left(\mathrm{M}^{2} \cdot \mathrm{K}\right)$, определяемый по уравнению (5):

$$
\alpha_{M}=\frac{Q}{F_{M}\left(\Delta t_{c p .2}-\Delta t_{c p . m}\right)},
$$

$F_{\mu}$ - площадь поверхности теплообмена, т.е. поверхность частиц, находящихся в теплообменном участке аппарата, ${ }^{2}$

$$
F_{M}=V \cdot a_{y \partial} .
$$

$V$ - объем теплообменного участка, м $^{3}$.

При рассмотрении стационарного процесса, для которого изменения температур газового и твердого компонентов во времени не изменяются, и при пренебрежении переносом теплоты теплопроводностью в газовой (воздушной) среде и в слое материала (как пренебрежимо малыми в сравнении с конвективным переносом), уравнения энергии (1) и (2) приводятся к следующему виду:

- для газового компонента:

$$
c_{2} \rho_{2} w_{2}^{\prime \prime} \frac{\partial t_{2}}{\partial x}=\alpha_{M} a\left(t_{2}-t_{m}\right) ;
$$

- для твердого компонента

$$
c_{m} \rho_{m}(1-\varepsilon) w_{m}^{\prime \prime} \frac{\partial t_{m}}{\partial x}=\alpha_{M} a\left(t_{2}-t_{m}\right) .
$$


Граничные условия:

$$
\begin{gathered}
x=0: t_{2}=t_{20} \\
x=L: t_{m}=t_{m 0} .
\end{gathered}
$$

Начальные условия:

$$
\tau=0: t_{2}=t_{20} ; t_{m}=t_{m 0} .
$$

Здесь $w_{m}^{\prime \prime}, w_{2}^{\prime \prime}-$ скорости слоя и воздуха в контактном теплообменнике, $L$ - высота слоя, $x$-продольная координата.

В результате решения системы уравнений (7), (8) с условиями (9), (10) получены зависимости для расчета локальных температур газового и твердого компонентов для противотока:

$$
\begin{gathered}
\operatorname{tg}(x)=C 1+C 2 e^{\frac{K 2(-K 4+K 3) x}{K 4 K 3}} \\
\operatorname{tm}(x)=\frac{K 4 C 1+C 2 e^{\frac{K 2(-K 4+K 3) x}{K 4 K 3}}}{K 4},
\end{gathered}
$$

где $C 1, C 2$ - коэффициенты, соответствующие заданным условием однозначности и определяемые зависимостями (13), (14):

$$
\begin{gathered}
C 1=\operatorname{tg} 0+\frac{K 4(\operatorname{tg} 0-\operatorname{tm} 0)}{-K 4+e^{K 5 \cdot L} K 3}, \\
C 2=-\frac{K 4(\operatorname{tg} 0-\operatorname{tm} 0)}{-K 4+e^{K 5 \cdot L} K 3} .
\end{gathered}
$$

Зависимости получены с помощью пакета прикладных программ MAPLE 15.

Для противотока учитывалось, что знак градиента температур имеет привязку к началу координат и как для воздуха, так и для дисперсного материала с ростом $x$ температура уменьшается. Коэффициенты $K 2, K 3, K 4$ представляют собой комбинации из задаваемых величин, характеризующих процесс:

$$
K 2=\alpha_{M} \cdot a_{y \partial},
$$

где $\alpha_{\mathrm{m}}-$ коэффициент межкомпонентного теплообмена, определяемый по рекомендациям [6], $a_{y d}$ - удельная поверхность частиц в единице объема.

$$
K 3=c_{2} \cdot \rho_{2} \cdot w .
$$

Зависимости для расчета температур при противотоке имеют следующий вид:

$$
\begin{gathered}
\operatorname{tg}(x)=C 1+C 2 e^{\frac{K 2(-K 4+K 3) x}{K 4 K 3}}, \\
\operatorname{tm}(x)=\frac{K 4 \cdot C 1+C 2 e^{\frac{K 2(-K 4+K 3) x}{K 4 K 3}} K 3}{K 4},
\end{gathered}
$$

Коэффициенты $C 1, C 2$ для противотока описываются следующими зависимостями:

$$
\begin{gathered}
C 1=\operatorname{tg} 0+\frac{K 4(\operatorname{tg} 0-\operatorname{tm} 0)}{-K 4+e^{K 5 \cdot L} \cdot K 3}, \\
C 2=-\frac{K 4(\operatorname{tg} 0-\operatorname{tm} 0)}{-K 4+e^{K 5 \cdot L} \cdot K 3},
\end{gathered}
$$

Сопоставление экспериментальных и расчетных данных на примере керамзита при противоточной схеме движения потоков показало удовлетворительную сопоставимость результатов [13].

Результаты расчета температур газового и твердого компонентов для противоточной и прямоточной схем движения представлены на рисунках 2 и 3 соответственно.

Расчеты проводились при таких параметрах: $\alpha_{M}=30 \mathrm{BT} /\left(\mathrm{M}^{2} \mathrm{~K}\right)$, высота слоя $l=0,52 \mathrm{м}$, порозность ( $\varepsilon=0,32$, средняя скорость движения плотного слоя керамзита $w_{m}=8,3 \cdot 10^{-4} \mathrm{M} / \mathrm{c}$, средняя скорость воздуха $w_{2}^{\prime}=1,3 \mathrm{M} / \mathrm{c}$.

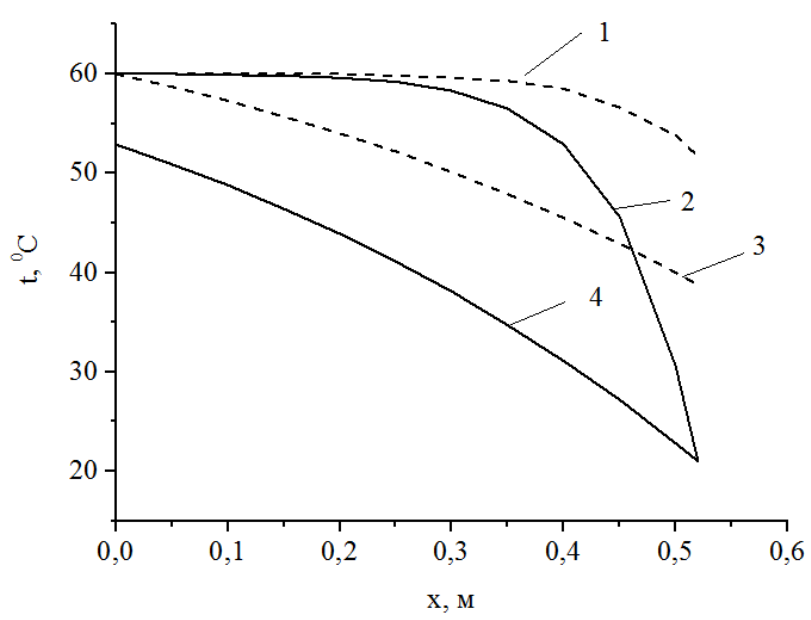

Рисунок 2 - Распределение температур газового $и$ дисперсного теплоносителей по высоте канала при противоточной схеме движения 1 -воздух-керамзит, 2 - керамзит 3 -воздух гравий, 4 - гравий

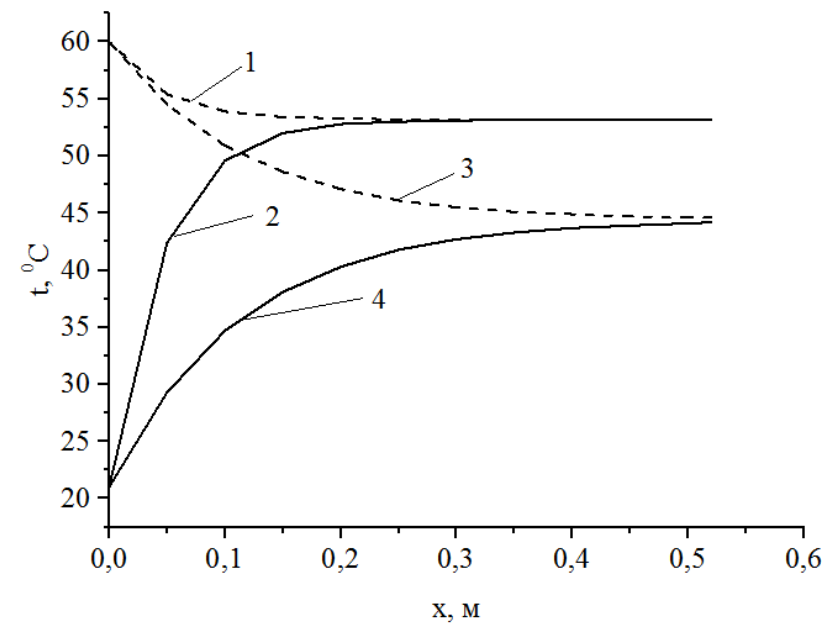

Рисунок 3 - Распределение температур газового и дисперсного теплоносителей по высоте канала при прямоточной схеме движения 1 -воздух-керамзит, 2 - керамзит 3 - воздух гравий, 4 - гравий

Результаты расчетов свидетельствуют об интенсивности теплообменных процессов при использовании дисперсного материала. Как для прямотока, так и для 
противотока практически полное выравнивание температур при нагреве керамзита происходит на участке канала длиной $L \approx 0,3$ м (для условий приведенного примера). Для гравия выравнивание температур наблюдается на высоте $L \approx 0,5$ м. Таким образом, расчетным путем представляется возможность определять оптимальные геометрические размеры теплообменного участка для различных рабочих параметров процесса. Результаты расчета также позволяют сделать вывод, что нагрев керамзита осуществляется значительно интенсивнее нагрева гравия, что связано с существенными различиями теплофизических свойств. Так, теплоемкость гравия $c_{z p}=875$ Дж/(кг·К), плотность $\rho_{z p}=2022$; теплоемкость керамзита $c_{\kappa}=840$ Дж/(кг·К), плотность $\rho_{\kappa}=662$. Полученные зависимости позволяют аналитически исследовать процессы теплообмена и могут быть предложены для оценки влияния геометрических и физических характеристик на эффективность работы теплоутилизаторов с дисперсной насадкой.

\section{3 Выводы}

Представленная двухкомпонентная модель стационарного теплопереноса в слое качественно верно описывает теплообмен между потоками газа и дисперсного материала. Полученные аналитические зависимости для расчета температур газового и твердого компонентов позволяют проводить расчетным путем оценку влияния различных факторов, таких как физические свойства газа и твердых частиц, скорости потоков, порозность слоя, на эффективность теплообмена между потоками, что может быть применено при поиске оптимальных параметров работы теплоутилизаторов контактного типа.

\section{Литература}

1. Бараков А. В. Исследование теплообмена в регенераторе с дисперсной насадкой [Текст] / А.В. Бараков, В.Ю. Дубанин, Д.А. Прутских // Энергосбережение и водоподготовка. 2007. №4. - С. 45-46.

2. Дехтярь Р. А., Сиковский Д. Ф., Горин А. В., Мухин В. А. Теплообмен в зернистом слое при умеренных числах Рейнольдса [Текст] // ТВТ. 2002. Т. 40, № 5. C. 748-755.

3. Jintang Li, David J. Mason. Application of the discrete element modelling in air drying of particulate solids// Technology - An International Journal, ISSN 0737-3937, 2002, vol.20, No.3, pp.255-282

4. Медведев В.Б., Субботин В.И., Мизонов В.Е., Елин Н.Н. Моделирование теплообмена между потоками газа и сыпучего материала при распределенной подаче газа [Текст] // Вестник ИГЭУ» Вып. 32008 г. c. 1-4

5. Горбис 3.Р., Календерьян В.А. Теплообменники с проточными дисперсными теплоносителями [Текст]. М: Энергия, 1975. -294 с

6. Буевич Ю.А. К теории переноса в гетерогенных средах [Текст] // Инж.-физ. журнал, 1988, т. 54, №5, с. 770-779

7. Антонишин Н.В., Лущиков В.В. Перенос тепла в дисперсных средах [Текст]//Исследование процессов переноса в дисперсных системах. Сборник научных статей. - Минск, 1984. с. 113-129

8. Botterill J.S. M., Denloye A.O. Gas convective heat transfer to packed and fluidized beds. A. I. Ch. E. Symp. Ser., 1978, v. 74, №176, p. 194-202

9. Нустров В.С., Сайфуллаев Б.Н. Метод эквивалентного уравнения в теории тепломассопереноса [Текст] // Инж.-физ. журнал, 1988, т. 54, №5, с. 779-786

10. Горбис 3.P. Теплообмен и гидромеханика дисперсных сквозных потоков [Текст], 1970, 424 с.

11. Аронов И.З., Пресич Г.А., Смирнов В.А. Анализ тепловой эффективности контактных теплоутилизаторов с промежуточным теплообменником [Текст] // Промышленная энергетика. 1986. № 1. С. 44-46

12. Календерьян, В. А. Тепломассоперенос в аппаратах с плотным дисперсным слоем [Текст] / В.А. Календерьян, И.Л. Бошкова // Монография. - К., 2011. - 184 с.

13. Бошкова І.Л., Солодка А.В. Исследование эффективности теплообмена в теплообменникахутилизаторах с гранулированной насадкой [Текст] // Аспекты регионального развития Матеріали міжнародної наукової конференції. - Молдова, Кишинів: Институт энергетики АН Молдовы, 2016. - С. 373-377.

Отримана в редакції 15.02.2017, прийнята до друку 25.04.2017

\title{
Mathematical Description of the Heat Exchange Process between Gas Flows and Disperse Material
}

\author{
A. V. Solodkaya ${ }^{1}$, I. L. Boshkova ${ }^{2}$ \\ Odessa National Academy of Food Technologies, 1/3, Dvoryanska Str., Odessa, 65082, Ukraine \\ e-mail: 1a_solodkaya@mail.ru, ${ }^{2}$ ira_boshkova@mail.ru \\ ORCID: ${ }^{1}$ http://orcid.org/0000-0002-4043-7667, ${ }^{2}$ http://orcid.org/0000-0001-5989-9223
}

Heat exchangers of contact type have a number of undoubted benefits in comparison with heat exchangers of surface type. Features of contact heat exchangers is the transfer of heat through direct contact of working bodies. The main advantages are the high intensity of heat transfer processes due to the developed heat exchange surface, the absence of the separation wall, the simplicity of design. The subject of the study is a heat recovery exchanger, in which heat exchange is carried out between gas flow and dispersed material. The importance of knowledge about the influence of individual factors and conditions on the temperatures distribution and heat exchange efficiency determine the need to construct the appropriate mathematical models. A two-component model describing heat exchange between gas and particle flows in a differential form is 
presented. As a result of its solution, analytical dependencies for calculating the temperatures of gas and solid components in the conditions of the steady-state regime for a direct-flow and countercurrent motion scheme were obtained. The results of calculations of component temperatures for two types of dispersed materials, gravel and expanded clay are presented. The obtained dependencies make it possible to study the processes of heat exchange analytically and can be proposed to assess the effect of geometric and physical characteristics on the efficiency of heat recovery exchangers, in which flows of gas and dispersible material interact.

Keywords: Heat exchanger; Dispersed material; Straight-flow; Countercurrent; Mathematical model; Temperature of components

\section{References}

1. Barakov, A.V., Dubanin, V.Yu., Prutskih, D.A. (2007) Issledovanie teploobmena $\mathrm{v}$ regeneratore $\mathrm{s}$ dispersnoi nasadkoi. Energosberezhenie $i$ vodopodgotovka, no.4, 45-46 (in Russian).

2. Dehtyar R.A., Sikovskiy D.F., Gorin A.V., Muhin V.A. (2002) Teploobmen v zernistom sloe pri umerennyh chislah Reynoldsa. TVT, vol. 40, no.5, 748-755 (in Russian); High Temperature, 40(5), 693-700. DOI: https://doi.org/10.1023\%2FA\%3A1020432619305

3. Jintang Li, David J. Mason (2002) Application of the discrete element modelling in air drying of particulate solids. Drying Technology, vol.20, no.3, 255-282. DOI: https://doi.org/10.1081/drt-120002542

4. Medvedev, V. B., Subbotin, V. I., Mizonov, V. E. Elin, N. N. (2008) Modelirovanie teploobmena mezhdu potokami gaza i sypuchego materiala pri raspredelennoi podache gaza. Vestnik IGEU, iss. 3, 1-4 (in Russian).

5. Gorbis, Z. R., Kalenderyan, V. A. (1975) Teploobmenniki s protochnymi dispersnymi teplonositelyami. Moskow, Energiia, 294 p. (in Russian).

6. Buevich, Yu. A. (1988) K teorii perenosa v geterogennykh sredakh. Inzh.-fiz. zhurnal, 54 (5), 770-779 (in Russian).
7. Antonishin, N. V., Lushchikov, V. V. (1984) Perenos tepla $\mathrm{v}$ dispersnykh sredakh. Issledovanie protsessov perenosa $v$ dispersnyh sistemah. Sbornik nauchnyh statey. Minsk, 113-129 (in Russian).

8. Botterill, J. S. M., Denloye, A. O. (1978) Gas convective heat transfer to packed and fluidized beds. AIChE Symp. Ser., 74 (176), 194-202.

9. Nustrov, V. S., Sayfullaev, B. N. (1988) Metod ekvivalentnogo uravneniia $\mathrm{v}$ teorii teplomassoperenosa. Inzh.-fiz. zhurnal, 54 (5), 779-786 (in Russian).

10. Gorbis, Z. R. (1970) Teploobmen i gidromehanika dispersnykh skvoznykh potokov, 424 p. (in Russian).

11. Aronov, I. Z., Presich, G. A., Smirnov, V. A. (1986) Analiz teplovoi effektivnosti kontaktnykh teploutilizatorov s promezhutochnym teploobmennikom. Promyshlennaya energetika, No.1, 44-46 (in Russian).

12. Kalenderyan, V. A., Boshkova, I. L. (2011) Teplomassoperenos v apparatakh s plotnym dispersnym sloem. Monografiia, 184 p. (in Russian).

13. Boshkova I. L., Solodka A. V. (2016)Issledovanie effektivnosti teploobmena $\mathrm{V}$ teploobmennikakhutilizatorakh s granulirovannoi nasadkoi. Aspekty regionalnogo razvitiya. Materialy mizhnarodnoi naukovoyi konferentsii. Moldova, Kishiniv: Institut energetiki AN Moldovy, 373-377 (in Russian). 